\title{
Disrupted-in-Schizophrenia 1 (DISC1) Is Necessary for the Correct Migration of Cortical Interneurons
}

\author{
André Steinecke, ${ }^{1 \star}$ Christin Gampe, ${ }^{1 \star}$ Christina Valkova, ${ }^{2}$ Christoph Kaether, ${ }^{2}$ and Jürgen Bolz ${ }^{1}$ \\ ${ }^{1}$ Institut für Allgemeine Zoologie und Tierphysiologie, Universität Jena, 07743 Jena, Germany, and ${ }^{2}$ Leibniz Institut für Altersforschung-Fritz Lipmann \\ Institut, 07745 Jena, Germany
}

\begin{abstract}
Disrupted-in-Schizophrenia 1 (DISC1) is a prominent susceptibility gene for major psychiatric disorders. Previous work indicated that DISC1 plays an important role during neuronal proliferation and differentiation in the cerebral cortex and that it affects the positioning of radial migrating pyramidal neurons. Here we show that in mice, DISC1 is necessary for the migration of the cortical interneurons generated in the medial ganglionic eminence (MGE). RT-PCR, in situ hybridizations, and immunocytochemical data revealed expression of DISC1 transcripts and protein in MGE-derived cells. To study the possible functional role of DISC1 during tangential migration, we performed in utero and ex utero electroporation to suppress DISC1 in the MGE in vivo and in vitro. Results indicate that after DISC1 knockdown, the proportion of tangentially migrating MGE neurons that reached their cortical target was strongly reduced. In addition, there were profound alterations in the morphology of DISC1-deficient neurons, which exhibited longer and less branched leading processes than control cells. These findings provide a possible link between clinical studies reporting alterations of cortical interneurons in schizophrenic patients and the current notion of schizophrenia as a neurodevelopmental disorder.
\end{abstract}

\section{Introduction}

Disrupted-in-Schizophrenia 1 (DISC1) is considered as one of the most compelling risk genes for schizophrenia, but also for other major psychiatric diseases. The biological functions attributed to the DISC1 protein are complex and highly diverse. For example, previous work suggested that DISC1 plays an important role during neuronal proliferation, differentiation, neurite outgrowth, synapse formation, as well as in the genesis and integration of new neurons in the adult hippocampus (for review, see Brandon et al., 2009). There are also several studies that report that DISC1 is a necessary component for the correct positioning of radially migrating cortical pyramidal neurons (Kamiya et al., 2005; YoungPearse et al., 2010).

We were interested whether DISC1 plays a role in the migratory behavior of cortical interneurons. There are distinct differences in the mode of migration of pyramidal neurons and interneurons. In contrast to cortical projection neurons, interneurons are generated in the medial ganglionic eminence (MGE), the caudal eminence, and the preoptic area (POA) of the basal telencephalon and perform a glia-independent long-range migration in a saltatory fashion (Marín and Rubenstein, 2001). They first extend a highly dynamic branched leading process and

Received Oct. 4, 2011; revised Nov. 24, 2011; accepted Dec. 1, 2011.

Author contributions: A.S., C.G., and J.B. designed research; A.S., C.G., C.V., and C.K. performed research; C.V. and C.K. contributed unpublished reagents/analytic tools; A.S. and C.G. analyzed data; J.B. wrote the paper.

We thank Dr. Morrison for providing the $\alpha \mathrm{GAPDH}$ antibody and Dr. Sawa for the murine DISC1 expression vector. We also thank Christine Raue for excellent technical assistance.

*A.S. and C.G. contributed equally to this work.

The authors declare no competing financial interests.

Correspondence should be addressed to Jürgen Bolz, Institut für Allgemeine Zoologie und Tierphysiologie, Universität Jena, Erbertstrasse 1, 07743 Jena, Germany. E-mail: jurgen.bolz@uni-jena.de.

DOI:10.1523/JNEUROSCI.5036-11.2012

Copyright $\odot 2012$ the authors $\quad 0270-6474 / 12 / 320738-08 \$ 15.00 / 0$ thereby scan for extracellular guidance cues that determine their migratory pathway (Valiente and Marín, 2010). Neurite elongation then stops and the nucleus moves forward, a process called nucleokinesis, and the migratory cycle starts again (Métin et al., 2006).

In this study, we first demonstrate that DISC1 transcripts and proteins are present in the MGE of embryonic day (E) 14.5 mouse embryos. We then manipulated DISC1 expression by RNA interference in individual MGE-derived interneurons to characterize cell-autonomous effects of DISC1. Results indicate that interneuron migration is severely delayed after DISC1 suppression. In addition to these migratory deficits, DISC1 knockdown also leads to distinct morphological changes of interneurons, suggesting a causal relationship between alterations in the cytoskeleton and the impaired migration behavior.

\section{Materials and Methods}

Mice. Animals used were timed pregnant C57BL/6 mice. The day of insemination was considered as E1. Mice were killed using peritoneal injection of $10 \%$ chloral hydrate. All animal procedures were performed in agreement with the institutional regulations of the University of Jena.

Plasmids. For DISC1 knockdown, the BLOCK-iT Pol II miR RNAi expression vector kit (Invitrogen) was used to design miRNA-expressing vectors targeting the sequence AGGCAAACACTGTGAAGTGCA, as described previously (Kamiya et al., 2005). As a control vector, we used a scrambled miRNA sequence that is predicted not to target any known vertebrate sequence. For rescue experiments, the DISC1 miRNA vector $(0.75 \mu \mathrm{g} / \mu \mathrm{l})$ was coelectroporated with either a pCMV6-XL5 encoding the human DISC1 transcript Lv (SC301729; OriGene Technologies) or a pCAX including mouse DISC1 (a gift from Dr. Sawa, Johns Hopkins University, Baltimore, MD), each at $2.25 \mu \mathrm{g} / \mu \mathrm{l}$. For overexpression of mouse DISC1 in NIH3T3, the DISC1 coding region from pCAX was cloned into pmRFP-C1.

Antibodies. The following primary antibodies were used: $\alpha$ DISC1 (1:50 for staining and 1:200 for Western Blotting; SC-47990, Lot \#2011; 
Santa Cruz Biotechnology), $\alpha$ GAPDH (1:1000; a gift from Dr. Morrison, Fritz Lipmann Institute, Jena, Germany), $\alpha$-Lim-homeobox-gene 6 ( $\alpha$ Lhx6; 1:200; SC-98607, Lot \#B-1609; Santa Cruz Biotechnology), $\alpha$ Calbindin (1:2000; Swant), and $\alpha$ GFP (1:1000; Invitrogen). Nuclei were stained with 4',6-diamidin-2-phenylindol (DAPI).

$R T-P C R$. Tissue from the MGE of E14.5 mice and tissue from the hippocampus of the mother were homogenized in trizol and RNA was isolated using chloroform and isopropyl alcohol. cDNA synthesis was performed using oligo dT primer. For RT-PCR, cDNA was amplified by the following primer pair: ACCCAGGATAGCCTGCCTGCA and ATCAGGTCACAGCCCGGCCA, using a HotStarTaq DNA Polymerase (Qiagen).

In situ hybridization. For in situ hybridization, a fragment of the mouse DISC1 (bases 1355-2031) was cloned into a pBluescript vector and DIGlabeled sense and antisense probes were generated. In situ hybridizations were performed as described previously (Zimmer et al., 2008).

Primary cell culture. For dissociated neurons from the MGE of E14.5 embryos, MGEs were dissected and collected in ice-cold HBSS supplemented with $0.65 \%$ glucose. After incubation with $0.025 \%$ trypsin in HBSS for $17 \mathrm{~min}$ at $37^{\circ} \mathrm{C}$, tissue was dissociated into single cells by trituration and filtered through a nylon gauze to remove cell aggregates. Neurons were cultured in DMEM [supplemented with $10 \%$ fetal bovine serum (FBS), $100 \mathrm{U} / \mathrm{ml}$ penicillin, $100 \mu \mathrm{g} / \mathrm{ml}$ streptomycin, and $0.4 \mathrm{~mm}$ L-glutamine] at $37^{\circ} \mathrm{C}$ and $5 \% \mathrm{CO}_{2}$ for $2 \mathrm{~d}$.

To approve the antigen specificity, ex utero electroporation was performed and neurons were extracted from the MGE as described above.

Fibroblast cell culture and Western blotting. NIH3T3 cells were grown in DMEM-F12 with 10\% FBS and 5\% penicillin/streptomycin under standard cell culture conditions and transfected with plasmids using Lipofectamine 2000 (Invitrogen) according to the manufacturer's protocol. Cells were lysed after $32 \mathrm{~h}$ in STEN buffer ( $150 \mathrm{~mm}$ sodium chloride, 50 mM Tris, 2 mm EDTA, and 0.2\% NP-40). Lysates were separated on $12 \%$ SDS-polyacrylamide gels and transferred to PVDF membranes. Membranes were blocked in TBS-T buffer $(300 \mathrm{~mm} \mathrm{NaCl}, 10 \mathrm{~mm}$ Tris, $\mathrm{pH}$ 7.6, and $0.1 \%$ Tween 20 ) for $30 \mathrm{~min}$ and then incubated with the primary antibody for $1 \mathrm{~h}$ at room temperature (RT) or overnight at $4^{\circ} \mathrm{C}$. After washing in TBS-T (four times for $8 \mathrm{~min}$ ), membranes were incubated for $1 \mathrm{~h}$ at RT with horseradish peroxidase-conjugated secondary antibodies. Membranes were washed again in TBS-T and the signal was detected using enhanced chemiluminescence.

Immunocytochemistry. Neurons and NIH3T3 were fixed in 4\% paraformaldehyde (PFA), washed with PBS containing $0.2 \%$ Triton X-100, and blocked for $1 \mathrm{~h}$ with $5 \%$ bovine serum albumin (BSA), $0.3 \%$ Tween 20 in PBS containing $0.2 \%$ Triton X-100. For immunostainings against Lhx6, cells were washed in heated citrate buffer and a blocking solution containing sodium azide was used ( $1 \%$ milk, $10 \%$ FBS, $1 \mathrm{mg} / \mathrm{ml} \mathrm{BSA}$, and $52 \mu \mathrm{g} / \mathrm{ml}$ sodium azide in PBS). Primary antibodies were applied overnight. After washing with PBS, secondary antibodies were incubated for $1 \mathrm{~h}$ followed by washes in PBS and DAPI staining.

Immunohistochemistry. For immunohistochemistry, freshly prepared cryosections $(18 \mu \mathrm{m}$ ) were fixed in $4 \%$ PFA in PBS for $30 \mathrm{~min}$ (for DISC1: $2 \%$ PFA, $10 \mathrm{~min}$ ) and washed in $0.2 \%$ Triton X-100 in PBS, blocked in $10 \%$ goat serum, $5 \%$ BSA, and $0.2 \%$ Triton X-100 in PBS for $1 \mathrm{~h}$, followed by the incubation of the primary antibodies overnight. For immunostainings against Lhx6, slices were cooked in citrate buffer and a special blocking solution containing sodium azide was used (1\% milk, $10 \% \mathrm{FBS}, 1 \mathrm{mg} / \mathrm{ml} \mathrm{BSA}$, and $52 \mu \mathrm{g} / \mathrm{ml}$ sodium azide in PBS). After washing, sections were incubated for $2 \mathrm{~h}$ with the secondary antibody followed by washing, incubating in DAPI, and embedding.

Ex utero electroporation. Brain hemispheres from E14.5 embryos were dissected in ice-cold sterile Krebs buffer (126 mM NaCl, $2.5 \mathrm{~mm} \mathrm{KCl}, 1.2$ $\mathrm{mm} \mathrm{NaH}_{2} \mathrm{PO}_{4}, 1.2 \mathrm{~mm} \mathrm{MgCl}, 2.1 \mathrm{mM} \mathrm{CaCl}_{2}, 25 \mathrm{~mm} \mathrm{NaHCO}_{3}$, and $11 \mathrm{~mm}$ glucose). Ex utero electroporation was performed as described previously (Yozu et al., 2005). In brief, $<1 \mu \mathrm{l}$ of a $1 \mu \mathrm{g} / \mu \mathrm{l}$ miRNA solution was pressure injected into the ventricular zone of the MGE, followed by electroporation of two pulses of $100 \mathrm{~ms}$ duration and $100 \mathrm{~V}$ using a BTX ECM 830 (Harvard Apparatus).

Outgrowth assay. After ex utero electroporation, MGE were dissected in methyl cellulose medium (MC) ( $0.4 \mathrm{~g}$ of methyl cellulose, 5\% FBS, $1 \%$ penicillin/streptomycin, $1 \% \mathrm{~L}$-glutamine, $0.1 \%$ glucose) and cut in $200 \times$ $200 \mu \mathrm{m}$ pieces using a tissue chopper. The MGE explants were precultured at $37^{\circ} \mathrm{C}$ and $5 \% \mathrm{CO}_{2}$ for $1 \mathrm{~h}$, embedded in chicken plasma that was cross-linked with thrombine, and cultured in MC medium for 2 DIV before fixation with $4 \%$ PFA in PBS. Before analyzing, we made certain that there were transfected cells in the explant and that the outgrowth of nontransfected cells was normal.

Slice cultures. After ex utero electroporation, hemispheres were embedded in 30\% low-melt agarose in PBS, cut into $250 \mu \mathrm{m}$ slices using a vibratome, and kept in Krebs buffer for postholding sections (supplemented with $10 \mathrm{~mm}$ HEPES, $1 \%$ penicillin/streptomycin, and $0.1 \mathrm{mg} / \mathrm{ml}$ gentamicin) on ice. Slices were transferred on membranes (Whatman) lying on neurobasal medium (supplemented with $2 \%$ B-27, $1 \%$ penicillin/streptomycine, $1 \%$ L-glutamine, and $0.5 \%$ glucose), and cultured for $3 \mathrm{DIV}$ at $37^{\circ} \mathrm{C}$ and $5 \% \mathrm{CO}_{2}$ before fixation with $4 \%$ PFA in PBS.

In utero electroporation. Timed pregnant female mice were treated with $4 \mathrm{mg} / \mathrm{kg}$ Carprofen for $30 \mathrm{~min}$ before deeply anesthetizing with a mixture of fentanyl $(0.05 \mathrm{mg} / \mathrm{kg})$, midazolam $(5 \mathrm{mg} / \mathrm{kg})$, and metedomidine $(0.5 \mathrm{mg} / \mathrm{kg})$. Afterward the uterine horns were exposed. Various constructs $(1 \mu \mathrm{l}$ of total volume, $1 \mu \mathrm{g} / \mu \mathrm{l}$ DNA plus $2 \%$ fast green to monitor the injection) were injected into the lateral ventricles of the embryos and electroporation (5 pulses at $40 \mathrm{~V}, 100 \mathrm{~ms}$ duration) was performed with a forceps electrode connected to a BTX ECM 830 (Harvard Apparatus). After $2 \mathrm{~d}$ in utero, brains were fixed in 4\% PFA in PBS and cryosections were made, followed by immunostaining against GFP.

Cell analysis. The transfected cells were scanned with a Zeiss LSM 510 and analyzed using ZEN 2009 software. Cells from at least three independent experiments were examined.

\section{Results}

\section{DISC1 is expressed in MGE-derived interneurons}

We first used RT-PCR on MGE tissue isolated from E14.5 embryos to examine the expression of DISC1 mRNA in this subdivision of the subpallium. As a positive control, we used adult hippocampus, where DISC1 remains highly expressed (Austin et al., 2004). RT-PCR showed similar signals in these two brain regions (data not shown). To analyze the spatial distribution of DISC1 transcripts in the telencephalon, we performed in situ hybridizations. As illustrated in Figure 1A, DISC1 mRNA was detected in the cerebral cortex with highest expression levels in the ventricular zone (VZ) and the subventricular zone (SVZ), consistent with previous findings (Ma et al., 2002; Austin et al., 2004). In the basal telencephalon, DISC1 transcripts were found in the VZ and SVZ of the lateral ganglionic eminence (LGE), MGE, and the POA (Fig. $1 A$ ).

Next, we wanted to examine the distribution of DISC1 at the protein level. We first tested the antigen specificity of the DISC1 antibody. For this, NIH3T3 fibroblasts were transfected with pmRFP-DISC1 or pmRFP-C1 (control) and immunostainings were performed. The exogenous DISC1 completely overlapped with the immunocytochemical signal (Fig. $1 E$ ). Analysis of the mean gray values showed a significant increase of the DISC1 signal in cells overexpressing DISC1 compared with cells expressing RFP only (Fig. $1 E^{\prime}$ ). In addition, we performed immunostainings with dissociated MGE cells that were transfected with either control miRNA or DISC1 miRNA (Fig. $1 F$ ) to downregulate endogenous DISC1. Compared with control transfected interneurons, there was a significant decrease in the DISC1 signal in cells transfected with DISC1 miRNA (Fig. $1 F^{\prime}$ ). Together, these findings indicate that the antibody reacts with both endogenous and exogenous DISC1 protein. We then used this antibody to perform Western blot analysis to quantify the knockdown efficiency of the DISC1 miRNA. Compared with control miRNA, the knockdown was not complete, but it suppressed $\sim 65 \%$ of both DISC1 isoforms known in mice (Fig. $1 G$ ). 


\section{DISC1 mRNA}

A

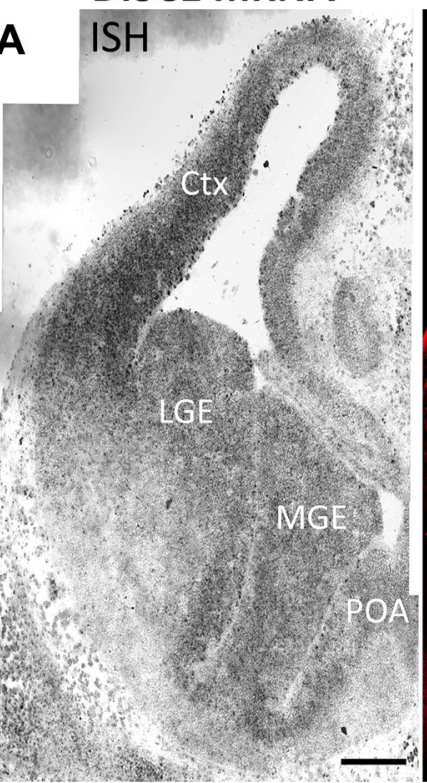

DISC1 protein

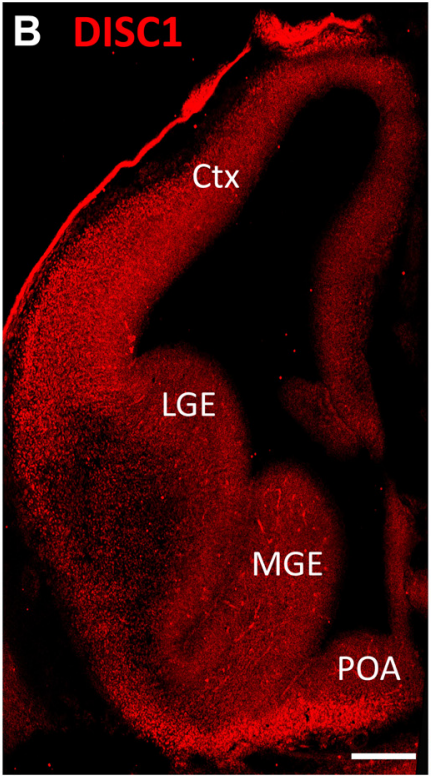

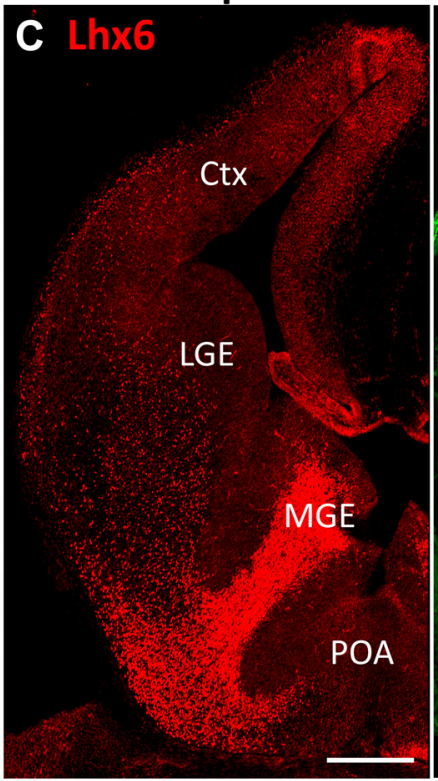

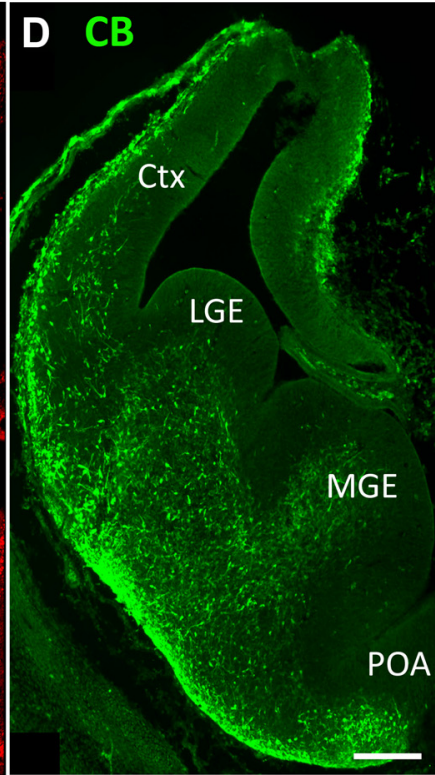

\section{antigene specificity of the DISC1 antibody}
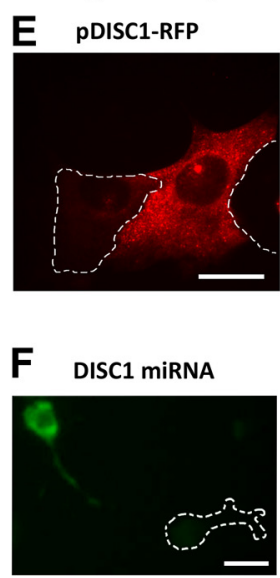

DISC1 immuno-

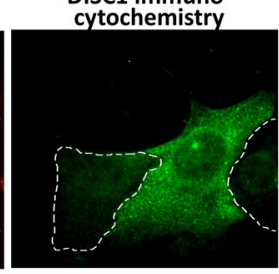

DISC1 immunocytochemistry

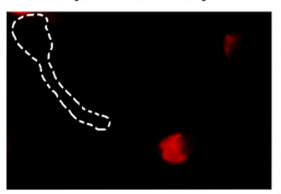

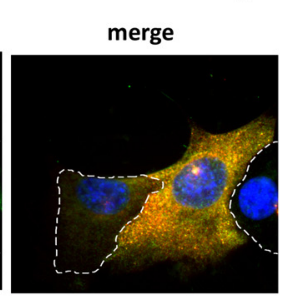

transmitted light

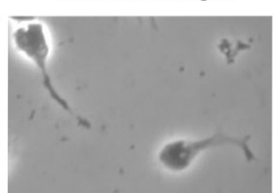

$\mathbf{E}^{\prime}$

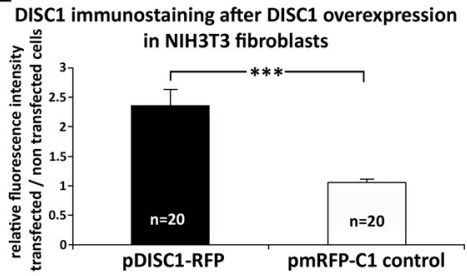

G DISC1 knock down control DISC1 MiRNA MiRNA

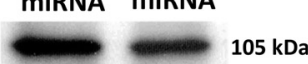

F' DISC1 immunostaining after DISC1 knock down

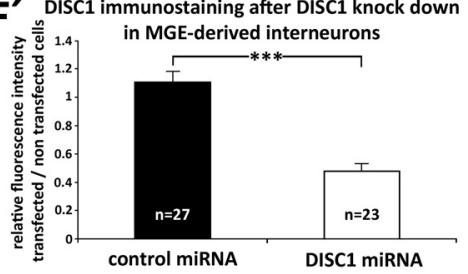

$\alpha$ DISC1

$\alpha$ GAPDH

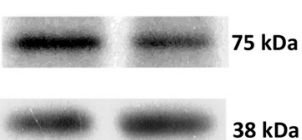

Figure 1. DISC1 mRNA and protein is expressed in E14.5 brains. A, In situ hybridization (ISH) demonstrates the expression of DISC1 mRNA in the cortex (Ctx), MGE, LGE, and P0A of E14.5 embryos. $\boldsymbol{B}-\boldsymbol{D}$, Immunohistochemistry with E14.5 cryosections of DISC1 (B), Lhx6 (C), and CB (D). The DISC1 signal overlaps with both markers for interneurons. Scale bars, $200 \mu \mathrm{m}$. $\boldsymbol{E}-\boldsymbol{F}$, Antigen specificity of the DISC1 antibody and knockdown of DISC1 by miRNA. Scale bars, $10 \mu \mathrm{m}$. E, Immunocytochemistry with NIH3T3 fibroblasts transfected with pmRFP-DISC1. $E^{\prime}$, Analysis of the mean gray value. There was a significant increase of the DISC1 signal in cells overexpressing DISC1 compared with cells expressing RFP only (RFP: $1.07 \pm 0.05$; DISC1-RFP: $2.36 \pm 0.28$; each 20 cells). $\boldsymbol{F}$, Immunocytochemistry on MGE-derived cells transfected with DISC1 miRNA. $F^{\prime}$, Comparison of the mean gray value. In interneurons transfected with DISC1 miRNA, the DISC1 signal was significantly decreased compared with control transfected cells (control miRNA: $1.11 \pm 0.07,27$ cells; DISC1: miRNA $0.74 \pm 0.05,23$ cells). G, Western blot to quantify the DISC1 knockdown efficiency in NIH3T3 fibroblasts. ${ }^{* *} p<0.001 ; t$ test. Error bars are SEM.

We then performed immunostainings of E14.5 cryosections. Results indicated DISC1 protein expression in the basal telencephalon concentrated in the VZ and the SVZ of the MGE, LGE, and POA (Fig. $1 B$ ), which is consistent with the findings of the in situ hybridization (Fig. 1A). In the SVZ of the basal telencephalon, the distribution of DISC1-immunoreactive cells overlapped with Lhx6-positive neurons (Fig. 1C), a transcription factor that is expressed in the vast majority of MGE neurons that migrate to the pallidum (Alifragis et al., 2004), and calbindin (CB)-positive neurons (Fig. $1 D$ ), another early marker for immature interneurons (Anderson et al., 1997). To confirm a coexpression of DISC1 with interneuron markers on a cellular level, we used dissociated MGE-derived cells to perform double immunostainings. As shown in Figure 2, first and second lane, DISC1 is expressed in Lhx6-positive as well as CB-positive cells.
Immunocytochemistry also allowed us to identify the subcellular distribution of DISC1. Previous studies demonstrated that the subcellular expression pattern of DISC1 is complex and cell type-specific. Endogenous DISC1 has been found within the nuclei of certain cell types (Sawamura et al., 2008), the cytoplasm, where it colocalizes with centrosomal proteins or F-actin (Morris et al., 2003), and also in the growth cones, the tips of growing axons (Shinoda et al., 2007). In MGE-derived interneurons, we found that DISC1 is consistently expressed in the cell body and in most cases also in the tips of cell processes, particularly in the filopodia of the leading process (Fig. $2 C$, arrow). In migrating interneurons, the cell body consists of a very thin layer of cytoplasm surrounding the nucleus. Confocal imaging of DISC1immunoreactive cells counterstained with the nuclear marker DAPI revealed that in the cell body, DISC1 was typically confined 

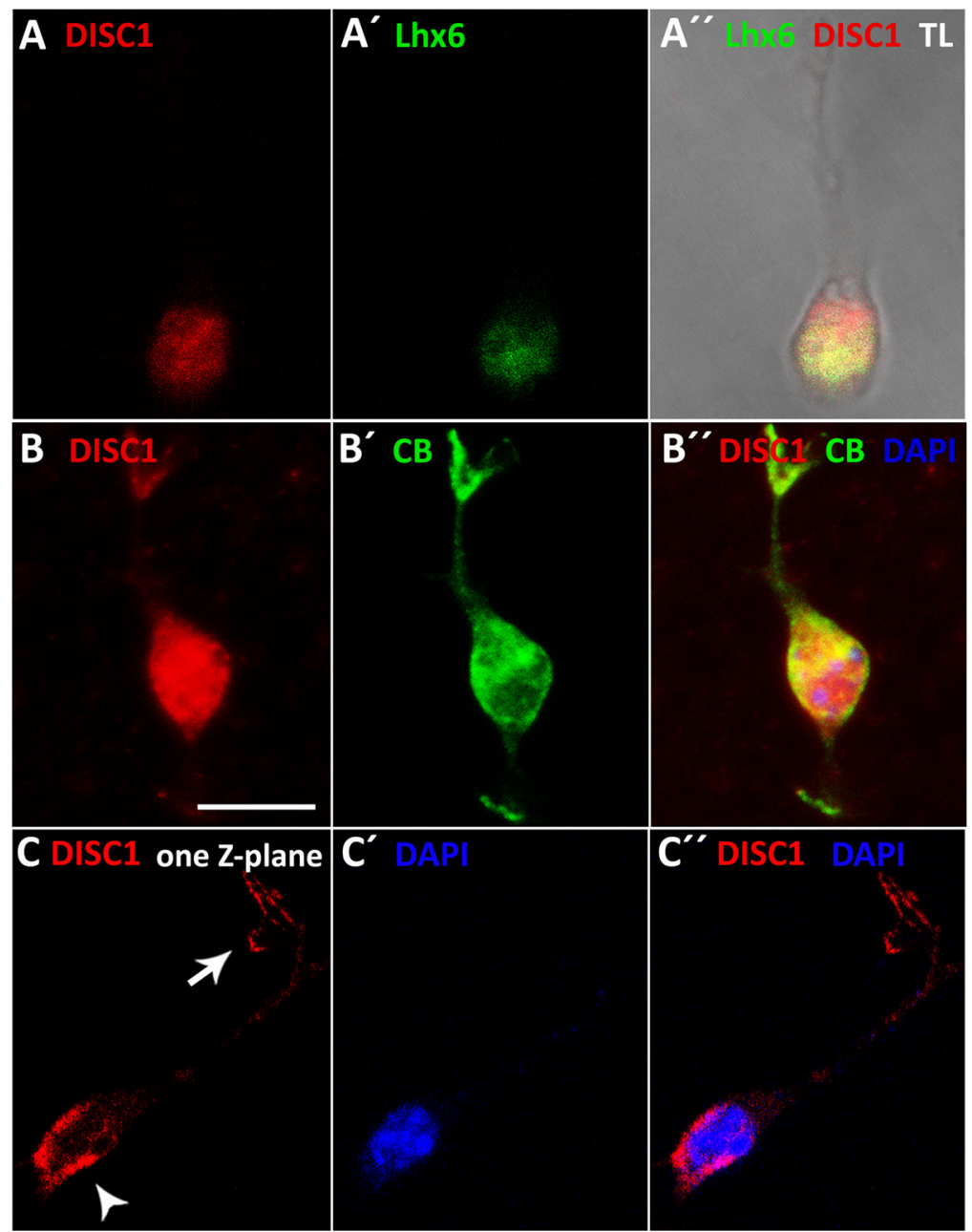

Figure 2. DISC1 is expressed in MGE-derived cortical interneurons. $\boldsymbol{A}, \boldsymbol{B}$, Dissociated cells from the MGE of E14.5 embryos show that DISC1 is coexpressed with Lhx6 $(\boldsymbol{A})$ and $C B(B)$. $\boldsymbol{C}$, Analysis of single Z-planes reveals that DISC1 is concentrated behind the nucleus (arrowhead). In addition, DISC1 is also concentrated in the tips of the leading process (arrow). Scale bar, $10 \mu \mathrm{m}, \mathrm{TL}$, Transmitted light.

to the cytoplasm of the cell body. In several cases, however, DISC1 was not evenly distributed in the cytoplasm around the cell nucleus, but was concentrated at the rear of the nucleus, opposite to the leading process (Fig. $2 C$, arrowhead). This subcellular distribution of DISC1 suggests that it might play a functional role in the migration of these neurons, for example by influencing nuclear translocation or the correct formation of leading processes.

\section{DISC1 knockdown leads to migration deficits in MGE-derived neurons}

To test the hypothesis that DISC1 is involved in the migration of MGE-derived cells, we first performed a cell migration assay combined with DISC1 RNA interference. For this, brain hemispheres of E14.5 embryos were ex utero electroporated with either a DISC1 miRNA vector or a control vector expressing control miRNA. Both vectors coexpressed EmGFP to visualize the transfected cells. After electroporation, small tissue blocks from the MGE were prepared and cultured for $2 \mathrm{~d}$ in a 3D matrix of crosslinked chicken plasma that allowed cell migration out of these explants. In this assay, downregulation of DISC1 with miRNA decreased the number of cells exiting the explants by $62 \%$ com- pared with cells transfected with control miRNA (from $4.7 \pm 0.6$ cells to $1.8 \pm 0.4$ cells per explant; Fig. $3 A, B, C$ ).

We next attempted to rescue the migration defects after DISC1 knockdown by coelectroporation DISC1 miRNA with expression constructs of human DISC1 (hDISC1), which are insensitive to the miRNA (Young-Pearse et al., 2010). As an additional control, we also coelectroporated DISC1 miRNA with expression constructs of murine DISC1 (mDISC1), which are susceptible to the miRNA. As illustrated in Figure 3C, after coexpression of DISC1 miRNA with mDISC1, the number of cells migrating out of MGE explants was still reduced compared with control conditions. In fact, there was no difference in the impaired migration between MGE cells transfected with DISC1 miRNA alone and cells cotransfected with mDISC1 plasmids, suggesting that DISC1 miRNA efficiently interferes with endogenous and exogenous murine DISC1. In contrast, coelectroporation of DISC1 miRNA with hDISC1 increased the number of interneurons leaving the explants by $67 \%$, from $1.80 \pm 0.18$ (DISC1 miRNA + mDISC1) to $3.02 \pm 0.27$ cells per explant (DISC1 miRNA + hDISC1). This indicates that hDISC1 significantly rescued the effects of DISC1 knockdown, although the rescue was not complete.

Next, we wished to examine directly how DISC1 knockdown affects interneuron migration in the basal telencephalon. It has been reported that ganglionic eminences can be specifically electroporated in utero by adjusting the angle of electrode paddles relative to the horizontal plane of the brain (Borrell et al., 2005). However, as others previously noticed (Gelman et al., 2009), we found that it is not possible to restrict in utero electroporation to MGE cells and most attempts result in transfections of cells either scattered throughout the entire subpallium or more or less focused clusters of transfected cells at random places in the basal telencephalon. Thus, when these brains are analyzed a few days after in utero electroporation, it is virtually impossible to know the exact origin of the transfected cells. We therefore used an ex utero electroporation approach, where brains were first removed from E14.5 embryos and miRNA plasmid solution was then pressure injected directly into the MGE before electroporation (Yozu et al., 2005). Adding Alexa 546 as a fluorescent marker to the miRNA solutions, we confirmed that the injections were spatially restricted to the MGE. We then prepared slice cultures from the ex utero electroporated brains, kept them for 3 DIV, and analyzed the distribution of the migration pattern of the transfected MGE cells (scheme Fig. 4A).

In slices prepared from ex utero electroporated brains, after 3 DIV, there was still a dense focus of transfected cells in the MGE, which made it difficult to quantify the exact number of these neurons. However, transfected MGE cells that had migrated to the LGE and transfected neurons that had continued their migra- 
tion to the cortex were clearly discernable. We therefore compared the number of cells transfected with DISC1 miRNA or control miRNA that had migrated from the MGE to the LGE with those that continued their migration to the cortex in each slice culture. The total number of labeled cells in the LGE and cortex was set as $100 \%$. After transfection with control miRNA, $58 \%$ of the labeled cells were found in the LGE while $42 \%$ of the labeled cells continued their migration to the cortex. In contrast, after transfection with DISC1 miRNA, 75\% were found in the LGE and only $25 \%$ reached the cortex (Fig. $4 B-D$ ). Thus, reduction of DISC1 in MGE neurons strongly impairs their tangential migration toward the cerebral cortex.

\section{DISC1 depletion changes the} morphology of migrating interneurons The migration of cortical interneurons is a complex process that goes along with distinct morphological changes. It is known that the leading process stops growing while the nucleus moves forward. When nucleokinesis was blocked or reduced, interneurons exhibited prolonged leading processes (Bellion et al., 2005). We therefore measured the length of the leading process of transfected cells in the outgrowth assay as well as in the slice cultures. In both assays, the length of the leading processes from DISC1-deficient cells were significantly longer than that of control transfected cells (Fig. 5A$D, G)$. Abnormally prolonged leading processes were not observed when DISC1 miRNA was cotransfected with hDISC1 (40.49 $\pm 1.67 \mu \mathrm{m}$ for control transfected cells; $35.65 \pm 1.76 \mu \mathrm{m}$ for DISC1 miRNA + hDISC1 transfected cells). As expected, there was no rescue effect for leading process length when cells were cotransfected with mDISC1 (Fig. $5 H$ ).

The leading processes of migrating interneurons are typically branched and it is generally assumed that they sample the environment for guidance cues (Martini et al., 2009). We therefore counted the number of side branches of cells transfected with DISC1 and control miRNA in both in vitro assays. We observed that control transfected cells branched significantly more often than DISC1-deficient cells. The highest number of side branches in cells transfected with DISC1 miRNA was three, while control transfected cells had up to six branches (Fig. 5A-D). On average, control transfected cells had $3.4 \pm 0.14$ branches; after DISC1 knockdown, the mean number of branches was reduced to $2.05 \pm 0.12(p<$ 0.001 ; Fig. $5 I$ ). After cotransfection of DISC1 miRNA with hDISC1, the number of sides branches significantly increased compared with transfections with DISC1 miRNA alone $(p<0.001$; Fig. 5J). However, cotransfections of DISC1 miRNA with mDISC1 did not increase the number of side branches observed after DISC1 knockdown (Fig. 5J). Thus, precise expression levels of DISC1 seem neces- sary for the formation of appropriate leading processes in migrating cortical interneurons.

To directly compare these in vitro results with the in vivo situation, we performed in utero electroporation of E14.5 embryos using control and DISC1 miRNA and examined the brains after $2 \mathrm{~d}$. As mentioned above, it is not possible to selectively transfect the MGE with this technique. We therefore restricted our morphometric analysis to cells that were still located in the MGE. Under these in vivo conditions, we could observe very similar morphological alterations as in the in vitro assays. MGE cells transfected with DISC1 miRNA had a leading process that was almost twice as long as in control transfected neurons $(55 \pm 7 \mu \mathrm{m}$ compared with $28 \pm 2 \mu \mathrm{m}$; Fig. $5 E-G)$. We also observed a modified branching pattern in DISC1-deficient cells after in utero electroporation. Over $90 \%$ of these cells had unbranched processes and the remaining cells had only one side branch. In contrast, approximately half of the control transfected cells had two or more side branches. The average number of branches was 

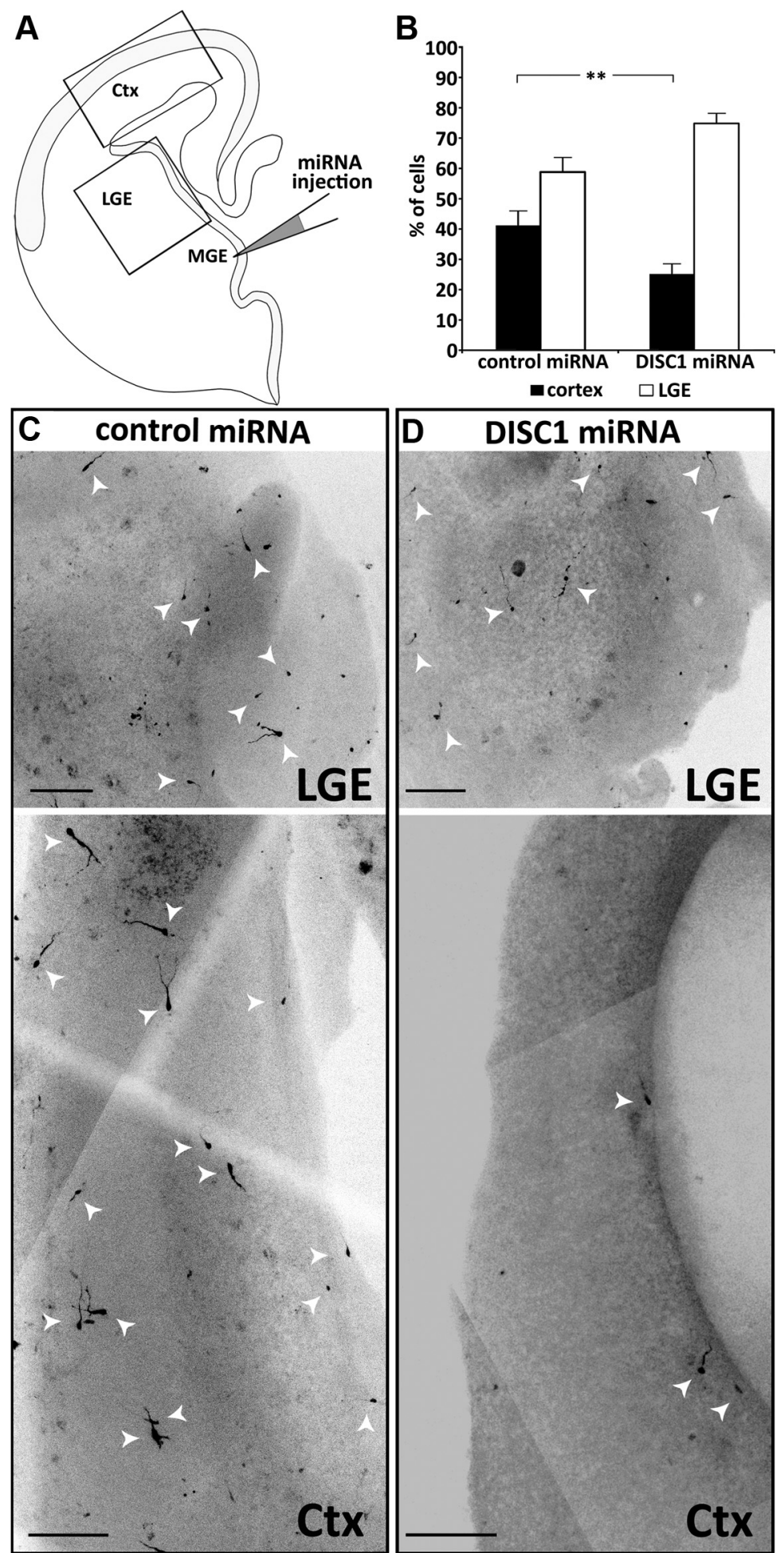

Figure 4. Focal DISC1 knockdown in the MGE leads to migration defects in slice cultures. $A$, Schematic drawing showing the injection of miRNA solution into the MGE of whole hemispheres followed by electroporation. The hemispheres were sliced and kept for 3 DIV. Rectangles indicate the regions of the photomicrographs shown below. B, Quantification of the number of cells which migrated to the LGE and cells that migrated further to the cortex (Ctx). Under control conditions, significantlymorecells reached the cortex compared with DISC1 knockdown slices (control miRNA:41.2 \pm 4.7\% cells in the cortex, $n=17$; DISC1 miRNA: $25.2 \pm 3.2$ cells in the cortex, $n=24 ; t$ test: $\left.{ }^{* *} p<0.01\right) .(-D$, Photomicrographs from cortex and LGE of control miRNA ( $)$ and DISC1 miRNA (D) transfected slices. Scale bars, $200 \mu \mathrm{m}$. Error bars are SEM.

\section{$2.7 \pm 0.2$ for control transfected cells and $1.1 \pm 0.1$ for DISC1} miRNA transfected cells $(p<0.001$; Fig. 5E-F, $I)$.

Together, the in vitro assays demonstrate that DISC1 knockdown leads to migratory deficits that correlate with specific changes in cell morphology. The fact that the same morphological alterations after DISC1 suppression also occurred in vivo strongly suggests that DISC1 is a necessary component for the correct migration of interneurons in the intact brain.

\section{Discussion}

In this study, we found that transcripts of the psychiatric illness risk gene DISC1 are expressed in the MGE, a major source of cortical interneurons. DISC1 protein was detected in MGE neurons that coexpress the early interneuron markers Lhx6 and $\mathrm{CB}$. In these neurons, DISC1 is concentrated in the tips of the leading process and its branches as well as in the somatic cytoplasm. Downregulation of DISC1 via miRNA increased the length of the leading process and at the same time decreased the number of side branches in MGEderived interneurons. Finally, cell migration assays revealed that DISC1 is a necessary component for the correct tangential migration of interneurons to the cerebral cortex.

Several previous studies have implicated DISC1 in regulating neuronal migration. For example, after in utero electroporation of DISC1 shRNA in cortical precursor cells in the ventricular zone, which gives rise to pyramidal neurons, transfected cells were retained in the intermediate zone and failed to migrate into the cortical plate (Kamiya et al., 2005). In contrast, knockdown of DISC1 in newly generated granule cells in the adult hippocampus resulted in an overextended migration (Duan et al., 2007; Enomoto et al., 2009; Kim et al., 2009). It has therefore been suggested that DISC1 does not directly mediate neuronal migration but rather relays positional cues to the migratory machinery (Duan et al., 2007). Consistent with this view, a recent study demonstrated that NMDA receptor antagonists in adult mice lead to an overextension in migration of newborn granule cells and a concomitant reduction in DISC1 expression (Namba et al., 2011). This overextended migration induced by NMDA receptors was rescued by exogenous expression of DISC1, demonstrating that extracellular signals can regulate DISC1 levels and thereby influence neuronal migration. In the present study, we found that after DISC1 suppression in the MGE, interneurons were able to migrate to the LGE and further to the cortex. However, the portion of DISC1deficient MGE cells that reached their final destination in the cortex was much lower than in control transfected MGE neurons. Thus, DISC1 does not seem to control the positioning of 


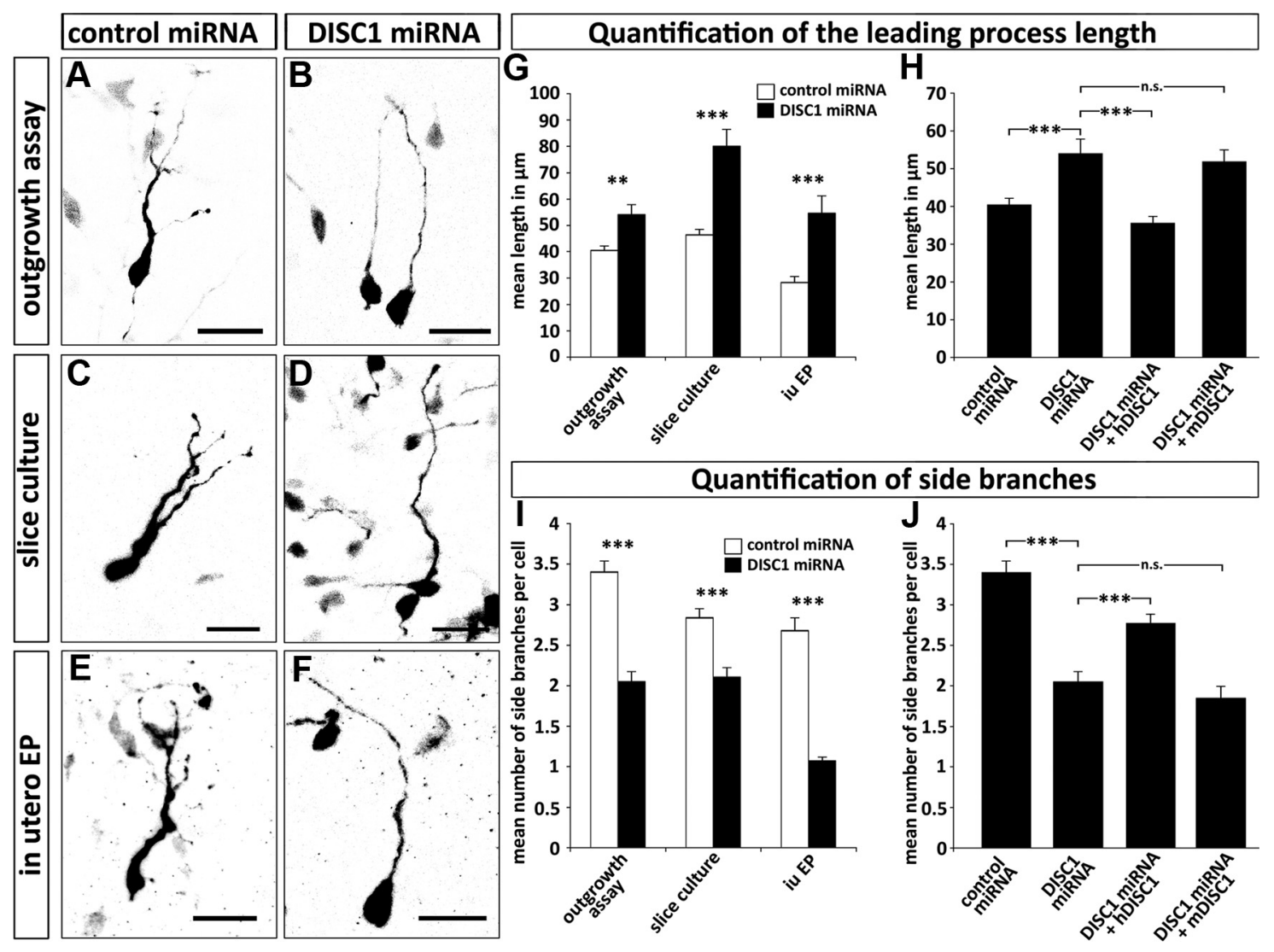

Figure 5. DISC1 knockdown leads to alterations in the morphology of MGE-derived interneurons. $A-F$, Photomicrographs of cells transfected with control miRNA or DISC 1 miRNA in outgrowth assays $(\boldsymbol{A}, \boldsymbol{B})$, slice cultures $(\boldsymbol{C}, \boldsymbol{D})$, and after in utero electroporation (iu EP) $(\boldsymbol{E}, \boldsymbol{F}) . \mathbf{G}-J$, Quantification of cell morphology. Under all experimental conditions, DISC1-deficient cells show longer, but less branched leading processes $(G, I)$. Cotransfection of miRNA with $\mathrm{mDISC}(1$ or hDISC1 in the outgrowth assay shows that the effect of DISC 1 knockdown can be rescued by a miRNA insensitive DISC1 $(\boldsymbol{H}, \boldsymbol{J})$. For statistical analysis, the means were compared with a Student's t test. Outgrowth assay: leading process length: control miRNA, $41 \pm 2 \mu \mathrm{m}$; DISC1 miRNA, $54 \pm 4 \mu \mathrm{m} ;$ DISC1 miRNA $+\mathrm{mDISC1}, 52 \pm 3 \mu \mathrm{m}$; DISC1 miRNA + hDISC1, $36 \pm 2 \mu \mathrm{m}$; number of side branches: control miRNA, $3.4 \pm 0.1 ;$ DISC1 miRNA, $2.1 \pm 0.1 ;$ DISC1 miRNA + mDISC1, 1.85 $\pm 0.15 ;$ DISC1 miRNA + hDISC1, $2.77 \pm 0.11$; control miRNA, $n=127$ neurites from 30 cells; DISC1 miRNA, $n=60$ neurites from 30 cells; DISC1 miRNA + mDISC1, $n=33$ neurites from 21 cells; DISC1 miRNA + hDISC1, $n=71$ neurites from 24 cells. Slice culture: leading process length: control miRNA, $46 \pm 2 \mu \mathrm{m}$; DISC1 miRNA, $80 \pm 6 \mu \mathrm{m}$; number of side branches: control miRNA, $2.8 \pm 0.1$; DISC1 miRNA, $2.1 \pm 0.1$; control miRNA, $n=92$ neurites from 30 cells; DISC1 miRNA, $n=66$ neurites from 30 cells. In utero electroporation: leading process length: control miRNA, $28 \pm 2 \mu \mathrm{m} ; \mathrm{DISC} 1 \mathrm{miRNA}, 55 \pm 7 \mu \mathrm{m}$; number of side branches: control miRNA, $2.7 \pm 0.2$; DISC1 miRNA, $1.1 \pm 0.1$; control miRNA $n=53$ neurites from 18 cells; DISC 1 miRNA, $n=28$ neurites from 26 cells). ${ }^{* * *} p<0.001 ; * * p<0.01 ; n . s . p>0.05$, $t$ test. Scale bars, $20 \mu \mathrm{m}$. Error bars are SEM.

cortical interneurons, but rather directly interferes with the ability of these neurons to migrate properly.

The dynamic behavior of different classes of migrating neurons has been described in detail (for review, see Ayala et al., 2007). The molecular mechanisms underlying these distinct cellular events, however, are still under debate. Alterations in the cytoskeletal proteins tubulin and actin, motor proteins of the dynein and kinesin family, and numerous regulatory proteins have been implicated to control the migration of diverse populations of neurons.

The subcelluar distribution of DISC1 in MGE neurons and the morphological alterations after DISC1 knockdown in these cells provide some clues how DISC1 might contribute to the migration of cortical interneurons. In migrating MGE neurons, before the initiation of nucleokinesis, actin, and the nonmuscle myosin II accumulate at the rear of the cell, forming a cup-like structure (Bellion et al., 2005). Blocking myosin II with belbbistatin reduced nuclear movement, suggesting that contraction of actinmyosin at the rear pushes the nucleus forward and presumably breaks adhesion and the trailing process at the cell rear. Since DISC1 is often localized in a cup-like shape at the trailing edge of MGE neurons, DISC1 might interact with F-actin and/or myosin motors. A colocalization of DISC1 with actin filaments and actin binding proteins has been reported previously (Morris et al., 2003).

We found that DISC1 is consistently expressed in the tips of the leading processes of MGE-derived interneurons. It has been suggested that dynein anchored in the membrane of the leading process is pulling microtubules attached to the centrosome and leading process. Recent work with migrating pyramidal neurons has shown that microtubule plus-end binding protein adenomatous polyposis coli (APC) is required to anchor microtubules to the distal end of the leading process (Asada and Sanada, 2010). If the localization of APC to the distal tips of the leading process is prevented, radial migration is impaired and the neurons exhibited longer leading processes. For migrating interneurons, where DISC1 is localized in the tips of the leading process, DISC1 might be involved in the attachment of microtubules to the distal ends of these neurites. This could explain why MGE neurons after DISC1 knockdown, in addition to their migratory deficits, also exhibit prolonged leading processes.

Obviously, the precise mechanisms how DISC1 is involved in interneuron migration remain to be determined. However, our results indicate that DISC1 has an impact on the migratory behavior of interneurons during early development that might lead to deficits in the number or composition of GABAergic neurons 
in the cortex. Dysfunctions of local GABAergic circuits have often been associated with the pathophysiology of schizophrenia (Benes and Berretta, 2001; Lewis et al., 2005). Thus, our findings support the notion that schizophrenia is a neurodevelopmental disease that may result at least in part from defects in neuronal integration (Lewis and Levitt, 2002).

\section{References}

Alifragis P, Liapi A, Parnavelas JG (2004) Lhx6 regulates the migration of cortical interneurons from the ventral telencephalon but does not specify their GABA phenotype. J Neurosci 24:5643-5648.

Anderson SA, Eisenstat DD, Shi L, Rubenstein JL (1997) Interneuron migration from basal forebrain to neocortex: dependence on $D l x$ genes. Science 278:474-476.

Asada N, Sanada K (2010) LKB1-mediated spatial control of GSK3beta and adenomatous polyposis coli contributes to centrosomal forward movement and neuronal migration in the developing neocortex. J Neurosci 30:8852-8865.

Austin CP, Ky B, Ma L, Morris JA, Shughrue PJ (2004) Expression of Disrupted-InSchizophrenia-1, a schizophrenia-associated gene, is prominent in the mouse hippocampus throughout brain development. Neuroscience 124:3-10.

Ayala R, Shu T, Tsai LH (2007) Trekking across the brain: the journey of neuronal migration. Cell 128:29-43.

Bellion A, Baudoin JP, Alvarez C, Bornens M, Métin C (2005) Nucleokinesis in tangentially migrating neurons comprises two alternating phases: forward migration of the Golgi/centrosome associated with centrosome splitting and myosin contraction at the rear. J Neurosci 25:5691-5699.

Benes FM, Berretta S (2001) GABAergic interneurons: implications for understanding schizophrenia and bipolar disorder. Neuropsychopharmacology 25:1-27.

Borrell V, Yoshimura Y, Callaway EM (2005) Targeted gene delivery to telencephalic inhibitory neurons by directional in utero electroporation. J Neurosci Methods 143:151-158.

Brandon NJ, Millar JK, Korth C, Sive H, Singh KK, Sawa A (2009) Understanding the role of DISC1 in psychiatric disease and during normal development. J Neurosci 29:12768-12775.

Duan X, Chang JH, Ge S, Faulkner RL, Kim JY, Kitabatake Y, Liu XB, Yang CH, Jordan JD, Ma DK, Liu CY, Ganesan S, Cheng HJ, Ming GL, Lu B, Song H (2007) Disrupted-In-Schizophrenia 1 regulates integration of newly generated neurons in the adult brain. Cell 130:1146-1158.

Enomoto A, Asai N, Namba T, Wang Y, Kato T, Tanaka M, Tatsumi H, Taya S, Tsuboi D, Kuroda K, Kaneko N, Sawamoto K, Miyamoto R, Jijiwa M, Murakumo Y, Sokabe M, Seki T, Kaibuchi K, Takahashi M (2009) Roles of disrupted-in-schizophrenia 1-interacting protein girdin in postnatal development of the dentate gyrus. Neuron 63:774-787.

Gelman DM, Martini FJ, Nóbrega-Pereira S, Pierani A, Kessaris N, Marín O (2009) The embryonic preoptic area is a novel source of cortical GABAergic interneurons. J Neurosci 29:9380-9389.

Kamiya A, Kubo K, Tomoda T, Takaki M, Youn R, Ozeki Y, Sawamura N, Park U, Kudo C, Okawa M, Ross CA, Hatten ME, Nakajima K, Sawa A
(2005) A schizophrenia-associated mutation of DISC1 perturbs cerebral cortex development. Nat Cell Biol 7:1167-1178.

Kim JY, Duan X, Liu CY, Jang MH, Guo JU, Pow-anpongkul N, Kang E, Song H, Ming GL (2009) DISC1 regulates new neuron development in the adult brain via modulation of AKT-mTOR signaling through KIAA1212. Neuron 63:761-773.

Lewis DA, Levitt P (2002) Schizophrenia as a disorder of neurodevelopment. Annu Rev Neurosci 25:409-432.

Lewis DA, Hashimoto T, Volk DW (2005) Cortical inhibitory neurons and schizophrenia. Nat Rev Neurosci 6:312-324.

Ma L, Liu Y, Ky B, Shughrue PJ, Austin CP, Morris JA (2002) Cloning and characterization of Disc1, the mouse ortholog of DISC1 (Disrupted-inSchizophrenia 1). Genomics 80:662-672.

Marín O, Rubenstein JL (2001) A long, remarkable journey: tangential migration in the telencephalon. Nat Rev Neurosci 2:780-790.

Martini FJ, Valiente M, López Bendito G, Szabó G, Moya F, Valdeolmillos M, Marín O (2009) Biased selection of leading process branches mediates chemotaxis during tangential neuronal migration. Development 136: $41-50$.

Métin C, Baudoin JP, Rakić S, Parnavelas JG (2006) Cell and molecular mechanisms involved in the migration of cortical interneurons. Eur J Neurosci 23:894-900.

Morris JA, Kandpal G, Ma L, Austin CP (2003) DISC1 (Disrupted-InSchizophrenia 1) is a centrosome-associated protein that interacts with MAP1A, MIPT3, ATF4/5 and NUDEL: regulation and loss of interaction with mutation. Hum Mol Genet 12:1591-1608.

Namba T, Ming GL, Song H, Waga C, Enomoto A, Kaibuchi K, Kohsaka S, Uchino S (2011) NMDA receptor regulates migration of newly generated neurons in the adult hippocampus via Disrupted-In-Schizophrenia 1 (DISC1). J Neurochem 118:34-44.

Sawamura N, Ando T, Maruyama Y, Fujimuro M, Mochizuki H, Honjo K, Shimoda M, Toda H, Sawamura-Yamamoto T, Makuch LA, Hayashi A, Ishizuka K, Cascella NG, Kamiya A, Ishida N, Tomoda T, Hai T, Furukubo-Tokunaga K, Sawa A (2008) Nuclear DISC1 regulates CREmediated gene transcription and sleep homeostasis in the fruit fly. Mol Psychiatry 13:1138-1148, 1069.

Shinoda T, Taya S, Tsuboi D, Hikita T, Matsuzawa R, Kuroda S, Iwamatsu A, Kaibuchi K (2007) DISC1 regulates neurotrophin-induced axon elongation via interaction with Grb2. J Neurosci 27:4-14.

Valiente M, Marín O (2010) Neuronal migration mechanisms in development and disease. Curr Opin Neurobiol 20:68-78.

Young-Pearse TL, Suth S, Luth ES, Sawa A, Selkoe DJ (2010) Biochemical and functional interaction of disrupted-in-schizophrenia 1 and amyloid precursor protein regulates neuronal migration during mammalian cortical development. J Neurosci 30:10431-10440.

Yozu M, Tabata H, Nakajima K (2005) The caudal migratory stream: a novel migratory stream of interneurons derived from the caudal ganglionic eminence in the developing mouse forebrain. J Neurosci 25:72687277 .

Zimmer G, Garcez P, Rudolph J, Niehage R, Weth F, Lent R, Bolz J (2008) Ephrin-A5 acts as a repulsive cue for migrating cortical interneurons. Eur J Neurosci 28:62-73. 